\section{Case Reports in Ophthalmology}

Case Rep Ophthalmol 2017;8:271-278

DOI: $10.1159 / 000475520$ Publisned onIIne: April 28, 2017
(C) 2017 The Author(s)

Published by S. Karger AG, Basel www.karger.com/cop

\title{
Branch Retinal Vein Occlusion, Macular Ischemia, and Intravitreal Anti-VEGF Therapy
}

\author{
Thomas Bertelmann ${ }^{a, b} \quad$ Hans Ulrich Frank $^{a}$ Hendrik Ansgar Fuchs $^{a}$ \\ Nicolas Feltgen ${ }^{b}$ \\ ${ }^{a}$ Belenus Eye Center Siegen, Siegen, Germany; ${ }^{b}$ Department of Ophthalmology, University \\ Medical Center Goettingen, Goettingen, Germany
}

\section{Keywords}

Branch retinal vein occlusion - Macular ischemia - Anti-VEGF - Corticosteroids - Intravitreal injection - Visual acuity $\cdot$ Central retinal thickness $\cdot$ Macular thickness $\cdot$ COMRADE

\begin{abstract}
Purpose: To report a case with ischemic macular edema (ME) due to an acute branch retinal vein occlusion (BRVO) which was treated with repeated intravitreal anti-VEGF injections. Methods: Retrospective case presentation. Results: A 66-year-old female patient was treated with repeated intravitreal anti-VEGF injections due to ischemic ME following an acute BRVO. Over a period of 2.5 years best corrected visual acuity increased from 0.06 to 0.6 (decimal notation) accompanied by a reduction in central retinal thickness from 546 to $292 \mu \mathrm{m}$. Overall 17 anti-VEGF injections were administered to treat repeated recurrence of ME. Macular ischemia did not worsen during this profound intravitreal anti-VEGF therapy. Conclusion: Intravitreal anti-VEGF therapy can be a beneficial treatment strategy even in ischemic ME following an acute BRVO.




\section{Case Reports in Ophthalmology}

\section{Introduction}

Branch retinal vein occlusion (BRVO) is the second most common retinal vascular disorder following diabetic retinopathy with an overall incidence of $0.5-1.2 \%[1,2]$. One major complication of BRVO is the development of a cystoid macular edema (ME) in more than $90 \%$ of the eyes affected, which in turn can cause severe visual disturbances and vision loss [2]. Despite the fact that BRVO and the consecutive ME can resolve spontaneously within 1 year in about $50 \%$ of patients, a prolonged ME can often hamper visual recovery [2]. Beside perfused, non-ischemic ME that can therapeutically be addressed with intravitreal anti-VEGF substances, corticosteroids, or focal laser treatment [3, 4], there is little information available about treatment success in ischemic cases. Recently, the first report of the 24-month, prospective, open-label, randomized, active-controlled, multicenter, phase IIIb study BRIGHTER ("Individualized Stabilization Criteria-Driven Ranibizumab versus Laser in Branch Retinal Vein Occlusion: Six-Month Results of BRIGHTER”) was published [5]. Herein, favorable results were described for eyes suffering from ischemic ME for the first time. To date it remains questionable if such treatment effects can also be observed in routine clinical care. The aim of this case report was to demonstrate the treatment success with intravitreally injected anti-VEGF in a patient suffering from ischemic ME due to an acute BRVO.

\section{Case Report}

A 66-year-old female patient, who complained about vision loss in her left eye, was initially seen in our department in April 2014. The conduced ophthalmologic examination revealed an acute BRVO in the inferior-temporal vein with consecutive ME development. Best corrected visual acuity (BCVA) in her left eye was $1 / 15$ (0.06; decimal notation). Fluorescein angiography (FAG) showed an acute BRVO with ischemia in the macular area and along the inferior temporal retinal arcade (Fig. 1).

Optical coherence tomography (OCT) analysis detected a central retinal thickness (CRT) of $546 \mu \mathrm{m}$ (Fig. 2). Except a beginning nuclear sclerosis of the lens, no further abnormalities were detected. Intraocular pressure was measured to be $17 \mathrm{~mm} \mathrm{Hg}$. The patient was sent to her family doctor for a complete medical workup and rheological therapy was conducted. Hypertension was diagnosed and subsequently treated.

Two weeks later intravitreal anti-VEGF therapy with bevacizumab (BEV) was initiated. After 3 injections, a marked increase in BCVA accompanied by a considerable CRT reduction was observed. A follow-up FAG in August 2014 and in September 2016 (Fig. 3) showed no changes in central retinal ischemia status; there was neither a decrease nor an increase in the size of the central ischemic retinal area. Retinal collaterals developed along the lower arcade as well as between the upper and lower arcade from month 4 on. A peripheral laser photocoagulation was advised in April 2014, but the patient did not give her consent for this procedure. One month later a recurrent cystoid ME was observed that was again successfully treated with 3 intravitreal BEV injections. After another ME relapse, therapy was switched to ranibizumab (RAN) and continued till October 2016. At the last visit, ME had again completely resolved and BCVA increased to 0.6 (Fig. 2). 


\section{Case Reports in Ophthalmology}

Case Rep Ophthalmol 2017;8:271-278

DOI: $10.1159 / 000475520$

c 2017 The Author(s). Published by S. Karger AG, Basel www.karger.com/cop

Bertelmann et al.: Branch Retinal Vein Occlusion, Macular Ischemia, and Intravitreal AntiVEGF Therapy

\section{Discussion}

The recently published BRIGHTER data showed the beneficial effect of intravitreally administered RAN to treat ischemic ME due to BRVO for the first time. Overall, eyes with ischemic ME gained BCVA of 14 letters within the 6-month study period. Furthermore, the comparison of treatment effects between ischemic and non-ischemic ME did not significantly differ [5]. This is surprising, because for ischemic ME the visual prognosis, irrespective of applied treatment modalities, was thought to be unfavorable [6]. In the presented case taken from routine clinical care, the functional improvement was comparable to the treatment success in the BRIGHTER study as shown by an increase of BCVA from 0.06 at the first visit to 0.6 almost 2.5 years later.

The favorable treatment success in our case might partially be attributed to the fact that the onset of BRVO was only 2 weeks before starting intravitreal anti-VEGF injections, and rheological therapy was immediately conducted. It is well known that recent occlusions respond better than older ones [7]. Older BRVOs can lead to profound alterations of the retinal structure which can be detected with OCT (e.g., discontinuation of the external limiting membrane, myoid and ellipsoid zone). This aspect had so far not been considered in the BRIGHTER analysis even though older BRVOs were included as well. However, the second OCT scan in our case taken in August 2014 showed no alterations of the outer retinal structures. Further research is indicated to clarify this as yet unanswered question.

Another important aspect refers to the treatment regimen. In our case 3 intravitreal anti-VEGF injections were performed until ME resolved, followed by observation and reinjections as soon as the recurrence of ME was detected. This might be the cause of a total of 5 episodes of a reappearance of ME. Applying a treat-and-extend scheme [7, 8] or switching to a dexamethasone implant [9] might be more favorable to avoid this zigzag pattern of CRT. At least with intravitreally injected steroids common complications like cataract worsening or an increase of intraocular pressure can occur, all of which we aimed to avoid [9].

Switching from BEV to RAN might have been too early in the presented case although previous research indicated that switching from one anti-VEGF substance to another might intensify the anti-VEGF effect. However, when reviewing the literature, there are to date no definite guidelines on when and how to switch drugs for eyes suffering from BRVO. Both, RAN compared to BEV, could resolve ME, and even after a total of 17 anti-VEGF injections and 2.5 years of therapy, no ME was evident in October 2016, and thus, anti-VEGF therapy was quite effective in this case.

Whereas focal laser treatments in cases of macular ischemia should be avoided [4], we recommended that a peripheral laser photocoagulation should be performed within the area of retinal ischemia. Unfortunately, this was refused by the patient. Recent reports indicate that a combination therapy including anti-VEGF and peripheral targeted laser treatment significantly reduces the injection frequency and stabilizes a dry macula, which was not achieved by anti-VEGF injections alone $[10,11]$. It might be hypothesized that with this combined treatment the frequently occurring ME relapses might have been avoided. So far, the literature does not provide a definite answer.

The most important aspect of this case is the observation of macular ischemia development during the profound anti-VEGF therapy. Herein, anti-VEGF blockade had no effect on the central area of non-perfused retinal tissue. These results are in accordance with a report by Rishi et al. [12] demonstrating that anti-VEGF treatment had no negative impact on ischemia development and a recently published review article about anti-VEGF treatment and macular ischemia [6]. Whereas Rishi et al. reported on their results after 1 single BEV injec- 
tion, our case clearly demonstrates the safety of anti-VEGF with regard to retinal ischemia development in eyes with ischemic BRVO, because overall 17 injections in a timeframe of 2.5 years were administered.

There are some reports published recently which show a prognostic value of OCT angiography and ischemic areas $[13,14]$. This new technique is still under debate and was not available in the present case.

\section{Conclusions}

As demonstrated with this case report and the data of the BRIGHTER study, intravitreally injected anti-VEGF drugs might be an excellent treatment option for ischemic macular edema due to an acute BRVO. Considerable BCVA gains and CRT reductions could be expected without increasing size and severity of central retinal ischemia in the short and long term. This seems to be plausible not only in phase III, randomized clinical trials, but also in routine clinical care. Further research in a routine clinical setting is indicated to confirm the results as described herein. Furthermore, the ischemia data taken from the COMRADE studies [15] are awaited to answer the question whether anti-VEGF or corticosteroids act similarly in such scenarios.

\section{Statement of Ethics}

The authors have no ethical conflict to disclose.

\section{Disclosure Statement}

Thomas Bertelmann has received funding for research and clinical trials from Alcon (USA), Alimera Sciences (USA), Allergan (Ireland), Bayer HealthCare (Germany), and Novartis (Switzerland), as well as consulting fees, honoraria, and travel reimbursement from Alcon (USA), Alimera Sciences (USA), Allergan (Ireland), Bayer HealthCare (Germany), and Novartis (Switzerland). He is a scientific staff member of the Department of Ophthalmology, University Medical Center Goettingen, Germany and was a Medical Advisor for Novartis Pharma GmbH (Nuremberg, Germany) from April 2015 till August 2016.

Nicolas Feltgen has received funds from Novartis, Allergan, Bayer, and Heidelberg Engineering.

\section{Funding Sources}

No funding was received for this research.

\section{References}

Rogers SL, McIntosh RL, Lim L, Mitchell P, Cheung N, Kowalski JW, Nguyen HP, Wang JJ, Wong TY:

Natural history of branch retinal vein occlusion: an evidence-based systematic review. Ophthalmology 2010;117:1094-1101.e1095. 
Bertelmann et al.: Branch Retinal Vein Occlusion, Macular Ischemia, and Intravitreal AntiVEGF Therapy

-2 Jaulim A, Ahmed B, Khanam T, Chatziralli IP: Branch retinal vein occlusion: epidemiology, pathogenesis, risk factors, clinical features, diagnosis, and complications. An update of the literature. Retina 2013;33:901-910.

-3 Pielen A, Feltgen N, Isserstedt C, Callizo J, Junker B, Schmucker C: Efficacy and safety of intravitreal therapy in macular edema due to branch and central retinal vein occlusion: a systematic review. PLoS One 2013;8:e78538.

4 Lam FC, Chia SN, Lee RM: Macular grid laser photocoagulation for branch retinal vein occlusion. Cochrane Database Syst Rev 2015;5:CD008732.

5 Tadayoni R, Waldstein SM, Boscia F, Gerding H, Pearce I, Priglinger S, Wenzel A, Barnes E, Gekkieva M, Pilz S, Monés J; BRIGHTER study group: Individualized stabilization criteria-driven ranibizumab versus laser in branch retinal vein occlusion: six-month results of BRIGHTER. Ophthalmology 2016;123:13321344.

6 Manousaridis K, Talks J: Macular ischaemia: a contraindication for anti-VEGF treatment in retinal vascular disease? Br J Ophthalmol 2012;96:179-184.

7 Freund KB, Korobelnik JF, Devenyi R, Framme C, Galic J, Herbert E, Hoerauf H, Lanzetta P, Michels S, Mitchell P, Monés J, Regillo C, Tadayoni R, Talks J, Wolf S: Treat-and-extend regimens with anti-VEGF agents in retinal diseases: a literature review and consensus recommendations. Retina 2015;35:14891506.

8 Rush RB, Simunovic MP, Aragon AV, Ysasaga JE: Treat-and-extend intravitreal bevacizumab for branch retinal vein occlusion. Ophthalmic Surg Lasers Imaging Retina 2014;45:212-216.

-9 Haller JA, Bandello F, Belfort R, Blumenkranz MS, Gillies M, Heier J, Loewenstein A, Yoon YH, Jacques ML, Jiao J, Li XY, Whitcup SM; OZURDEX GENEVA Study Group: Randomized, sham-controlled trial of dexamethasone intravitreal implant in patients with macular edema due to retinal vein occlusion. Ophthalmology 2010;117:1134-1146.e3.

10 Tomomatsu Y, Tomomatsu T, Takamura Y, Gozawa M, Arimura S, Takihara Y, Inatani M: Comparative study of combined bevacizumab/targeted photocoagulation vs bevacizumab alone for macular oedema in ischaemic branch retinal vein occlusions. Acta Ophthalmol 2016;94:e225-230.

-11 Rehak M, Tilgner E, Franke A, Rauscher FG, Brosteanu O, Wiedemann P: Early peripheral laser photocoagulation of nonperfused retina improves vision in patients with central retinal vein occlusion (results of a proof of concept study). Graefes Arch Clin Exp Ophthalmol 2014;252:745-752.

$\$ 12$ Rishi P, Raka N, Rishi E: Analysis of potential ischemic effect of intravitreal bevacizumab on unaffected retina in treatment-naïve macular edema due to branch retinal vein occlusion: a prospective, interventional case-series. PLoS One 2016;11:e0162533.

13 Wons J, Pfau M, Wirth MA, Freiberg FJ, Becker MD, Michels S: Optical coherence tomography angiography of the foveal avascular zone in retinal vein occlusion. Ophthalmologica 2016;235:195202.

14 Kang JW, Yoo R, Jo YH, Kim HC: Correlation of microvascular structures on optical coherence tomography angiography with visual acuity in retinal vein occlusion. Retina 2016, Epub ahead of print.

15 Pielen A: Development of best corrected visual acuity in RVO patients as a function of central avascular area and peripheral retinal non-perfusion: secondary analysis of the COMRADE trials. EURETINA 2016, Copenhagen. 
Case Reports in
Ophthalmology
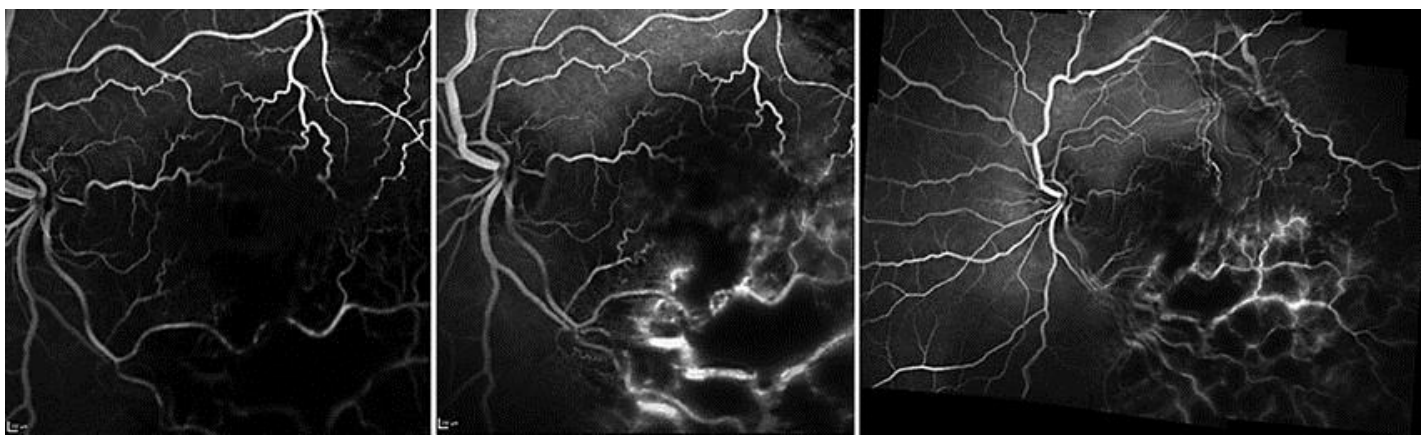

Fig. 1. Fluorescein angiography showed profound macular and peripheral ischemia in the early phase (left), in the middle phase (middle), and in the late phase (right, composite view). VEGF Therapy 
Bertelmann et al.: Branch Retinal Vein Occlusion, Macular Ischemia, and Intravitreal AntiVEGF Therapy
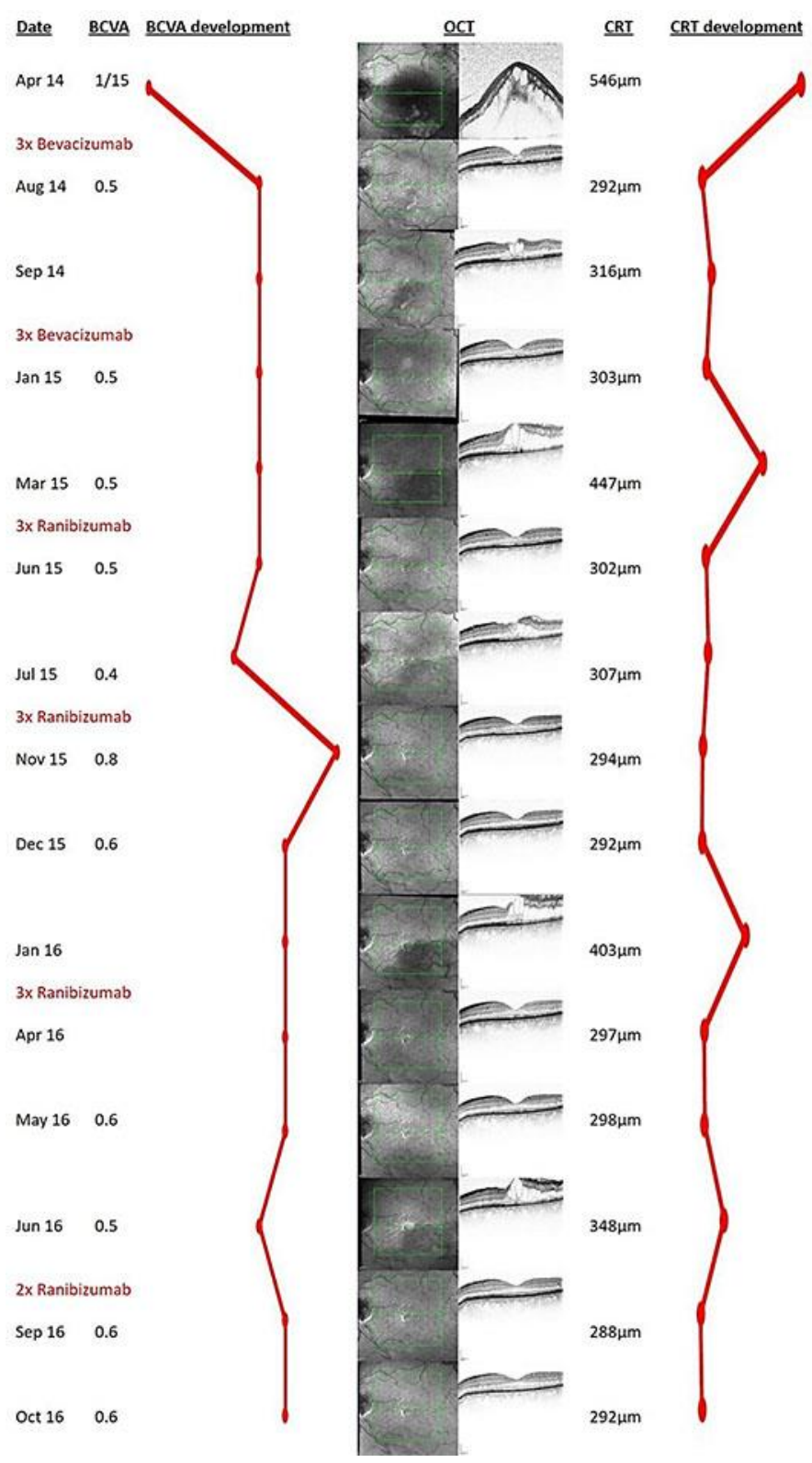

Fig. 2. Intravitreal injections, development of BCVA and CRT, and corresponding OCT images between April 2014 and October 2016. 
Case Reports in
Ophthalmology
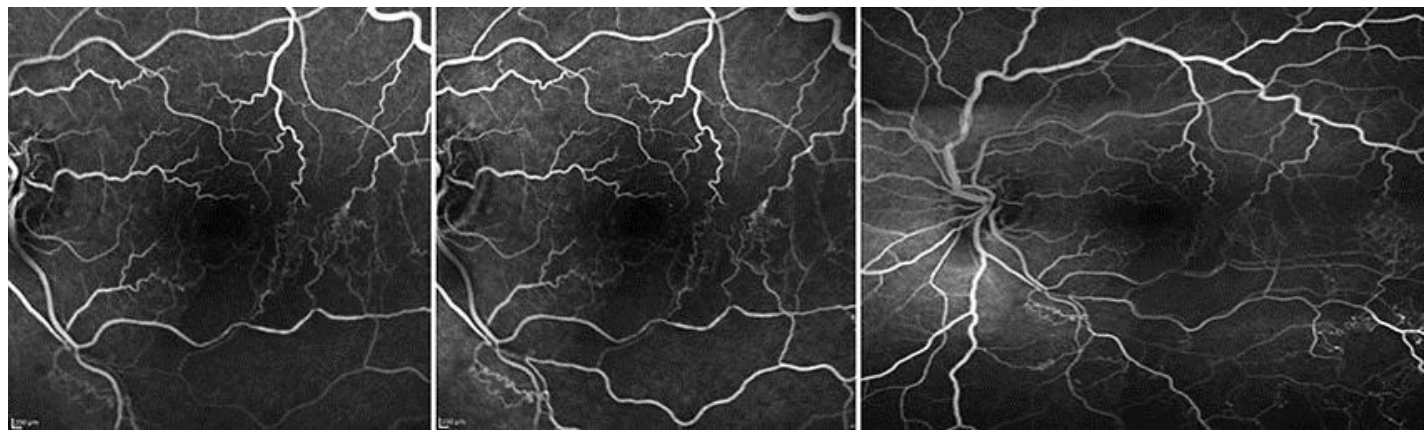

Fig. 3. Follow-up fluorescein angiography showed profound macular and peripheral ischemia in the early phase (left), in the middle phase (middle), and in the late phase (right, composite view).

Bertelmann et al: Branch Retinal Vein Occlusion, Macular Ischemia, and Intravitreal AntiVEGF Therapy 